\title{
Clinical Importance of Dental Implants Penetration in Maxillary Sinus. Literature Review

Simões $\mathrm{AF}^{1}$; Ragucci $\mathrm{GM}^{1}$; Ortiz-Puigpelat $\mathrm{O}^{1}$; Gargallo-Albiol J11 \& Hernández-Alfaro $\mathrm{F}^{1}$

\section{Abstract}

Background: The posterior edentulous maxilla usually presents a limited amount of bone volume due to the normal resorption of the alveolar ridge and the sinus pneumatization, which can be a complication for the implant placement in that area.

Objective: The purpose was to review the effect of dental implants penetrated on maxillary sinus, as well as, their success rate.

Material \& Methods: An electronic search was performed in Pubmed combining the following inclusion terms: "penetrant* implant" or "maxillary sinus" or "sinus membrane" or "sinus elevation" or "sinus complication".

Results: A total of 53 titles were indentified, from which 44 were excluded, ending with only 6 articles to proceed with the review. Three articles were studies base on animals and 4 in humans.

Conclusions: The success rate of penetrated implants in maxillary sinus is high, without any kind of complications, especially in the cases where the sinus intrusion is less than $2 \mathrm{~mm}$ and the implant is completely covered by Schneider's membrane.

\section{Background and Aim}

The posterior edentulous maxilla usually presents a limited amount of bone volume due to the normal resorption of the alveolar ridge and the sinus pneumatization ${ }^{1,2}$, which can be a complication for the implant placement in that area $^{3}$.

Some alternatives for this situations could be the sinus lift procedures with the placement of graft materials, which is considered the gold standard approach. However, variables of cost, healing time, postoperative pain, swelling, membrane perforation and graft failure can occur ${ }^{4}$. A second option could be the rehabilitation with short implants and the zygomatic implants have been used ${ }^{5}$.

Sometimes placement of dental implants in the posterior maxillary area with limited bone height can accidentally expose the apex of the implants into the sinus cavity ${ }^{1}$. Therefore, it can contribute to increase the implant failures and sinus infection ${ }^{2}$, the aim of the present review is to evaluate the effect of dental implants penetrated on maxillary sinus and their success rate.

\section{Methods and Materials}

An electronic search was performed in Pubmed combining the following inclusion terms: "penetrant* implant" or "maxillary sinus" or "sinus membrane" or "sinus elevation" or "sinus complication".

A total of 53 titles were indentified, from which 44 were excluded, ending with only 6 articles to proceed with the review. Three articles were studies base on animals and 4 in humans.

\begin{tabular}{|l|l|}
\hline \multicolumn{2}{c}{ Results } \\
\hline \multicolumn{1}{|c|}{ Animal Study } & \multicolumn{1}{c}{ Human Study } \\
\hline Zhong et al 2013 & Kim et al 2013 \\
\hline Jung et al 2006 & Abi Naim et al 2013 \\
\hline Branemark et al 1984 & Trabizi et al 2012 \\
\hline & Branemark et al 1984 \\
\hline
\end{tabular}

The study of Zhong et al 2013 on dogs found that the sinus intrusion of implants up to $2 \mathrm{~mm}$ did not create complications, seeing that the sinus membrane healed around the implants. On the other hand when the perforation was $3 \mathrm{~mm}$ or more, Schneider's membrane did not cover the implants exposing the apical section of the implant to debris accumulation and enhacing patients predisposition to sinusitis. Similar healing findings were seen by Junge et al regarding penetration up to $2 \mathrm{~mm}$, although if intrusion was 4 or $8 \mathrm{~mm}$, complete membrana coverage was not achieved.

Abi Najm et al 2013 performed human study in 83 implants with a sinus intrusion of less than $3 \mathrm{~mm}$. In a 9.9 year follow up they did not find clinical or radiographical signs of sinusitis in any of the patients and osteointegration was achieved in all the implants.

In a retrospective study Kim et al 2013 found a 100\% success rate in 87 implants penetrated into the sinus from 1 to $5 \mathrm{~mm}$. Only 3 patients $(7 \%)$ had a complication of nasal bleeding. They also found a osseous gain of $3,4 \mathrm{~mm}$ when implants were placed in a crest with $4,99 \mathrm{~mm}$ of height.

Tabrizi et al in his retrospective study did not find any sign nor sintoms of sinusitis. The 18 implants where perfectly osteointegrated after 12 months of follow-up.

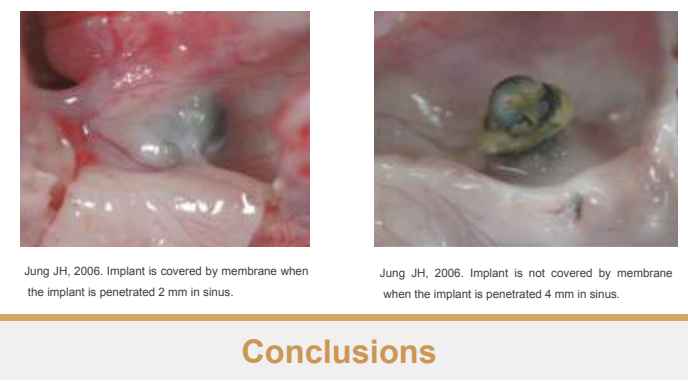

We can concluded that the success rate of penetrated implants in maxillary sinus is high, without any kind of complications, especially in the cases where the sinus intrusion is less than $2 \mathrm{~mm}$ and the implant is completely covered by Schneider's membrane.

However, it is necessary more randomized clinical studies in humans, once the present literature are almost animal or retrospective human studies.

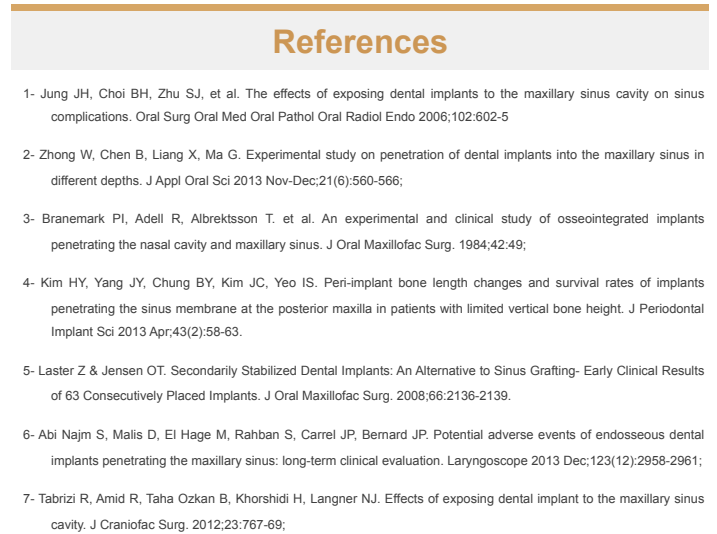

